\section{H1N1-associated sinus node dysfunction}

A 51-year-old previously healthy man presented to the emergency room following an episode of presyncope. He had a history of fever, pleuropericardial chest pain and dyspnoea accompanied by important myalgia evolving over several days. His past medical history was negative for any cardiovascular or pulmonary disease, and he was a lifelong non-smoker. He was previously very active with an excellent exertional capacity. His physical exam showed no frank heart failure, and he was haemodynamically stable.

Laboratory results showed increased inflammatory markers including an erythrocyte sedimentation rate of $35 \mathrm{~mm} / \mathrm{h}$ (normal $2-26 \mathrm{~mm} / \mathrm{h}$ ) and a C-reactive protein of $17 \mathrm{mg} / \mathrm{L}$ (normal $0.2-$ 7.6). There was also a leucopenia at $3.7 \times 10^{9} / \mathrm{L}$ (normal $4.0-11.0$ ) with a subtle lymphopenia of $0.9 \times 10^{9} / \mathrm{L}$ (normal $1.0-4.0$ ) and a light thrombocytopenia of $114 \times 10^{9} / \mathrm{L}$ (normal 150-400). Tropononin Twas elevated at $0.15 \mu \mathrm{g} / \mathrm{L}$ (normal <0.05).

The ECG showed sinus rhythm with complete left bundle branch block (LBBB) (figure 1A). Echocardiography demonstrated a mildly dilated left ventricle with reduced ejection fraction (35\%). Cardiac MRI was suggestive of myocarditis with preserved myocardial wall thickness. PCR analysis of nasopharyngeal swab confirmed influenza A H1N1 pandemic strain of 2009 (H1N1pdm2009) infection.
In hospital, the patient had repeat symptomatic sinus pauses of up to $6 \mathrm{~s}$ (figure 1B). Sinus node dysfunction and the LBBB persisted after several days of observation and despite the absence of myocardial oedema at the level of the sinus node and the mitro-aortic junction and normalisation of all inflammatory biomarkers. Therefore, we finally performed a pacemaker implantation (dual-chamber Medtronic Adapta ADDRL1; Medtronic, Indianapolis, Indiana, USA; St. Jude Tendril 2088TC leads were implanted in the atrium and the ventricle, St. Jude Medical, St. Paul, Minnesota, USA). The device was programmed to an AAIR-DDDR mode with a base rate of $50 \mathrm{bpm}$. Standard medication for heart failure was initiated, and the patient was discharged home with full symptomatic recovery. A repeat echocardiogram 3 months later showed an improved left ventricular ejection fraction of $45 \%$. A device interrogation at the same time showed only partial resolution of the sinus node dysfunction. The patient was paced in the atrium for $51 \%$ of the time but only $0.6 \%$ of the time in the ventricle.

Although H1N1pdm2009 associated atrioventricular (AV) nodal block is described, sinus node dysfunction has not been previously reported in this pandemic. ${ }^{1}$ Sinus arrest may result in severe bradycardia and significant morbidity and mortality. We can only speculate on the underlying pathomechanism of the myocarditis-associated sinus node dysfunction in our case. Also, it is unclear if a true sinus node dysfunction or a sinoatrial exit block of the affected surrounding myocardium caused the pauses in our patients. Interstitial inflammatory oedema has been described to cause potentially reversible conduction abnormalities at the level of the $\mathrm{AV}$ node, ${ }^{2}$ but this has not been
Figure 1 (A) Twelve-lead ECG showing sinus rhythm with a complete left bundle branch block. (B) Telemetry rhythm strip showing initial sinus rhythm with progressive slowing of the heart rate followed by an intermittent sinus pause of $6 \mathrm{~s}$.

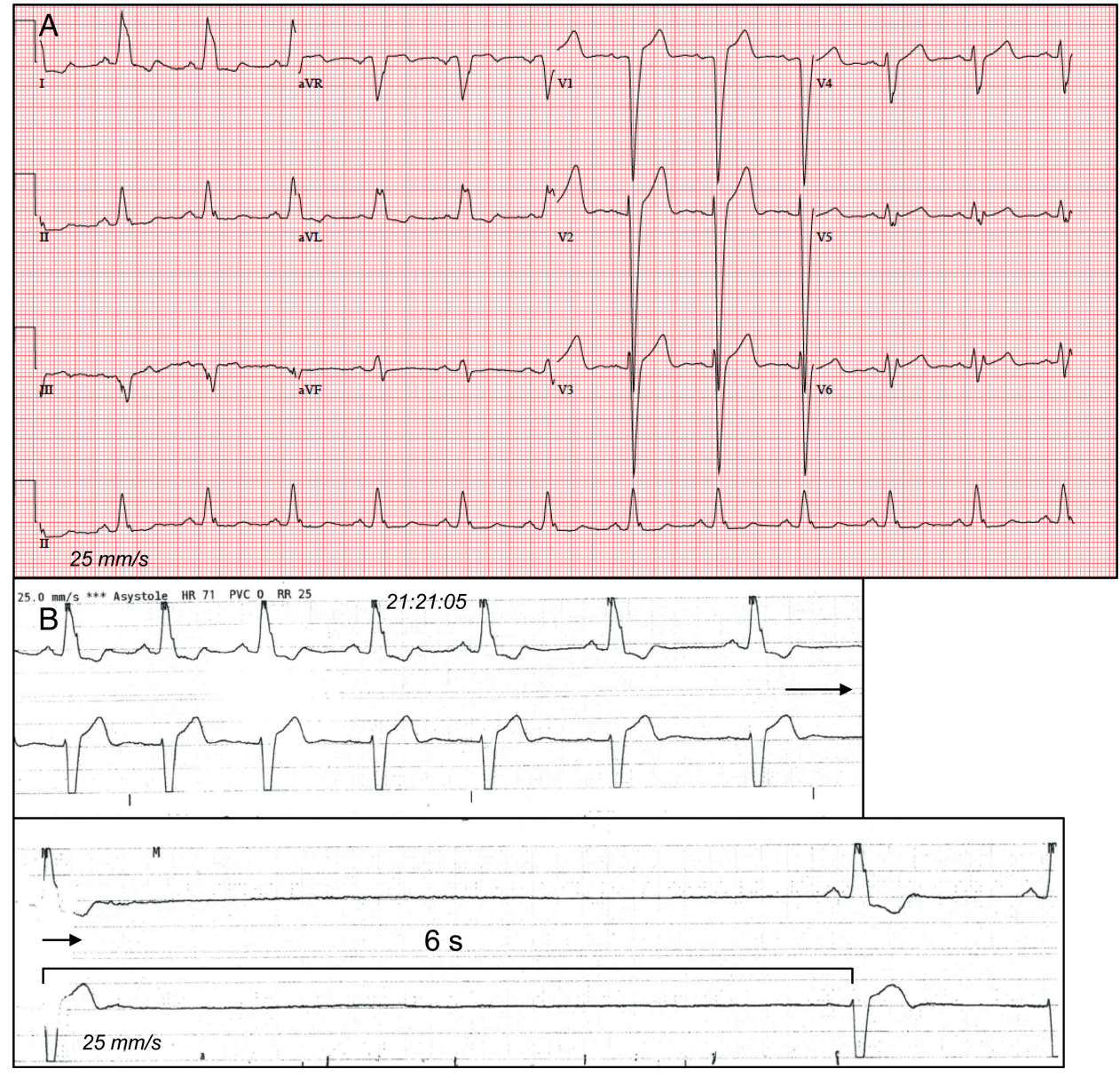

Steinberg C, et al. Heart Asia 2015;7:16-17. doi:10.1136/heartasia-2014-010522 
shown for the sinus node so far. Sinus node dysfunction and LBBB persisted in our patient even after resolution of all clinical and biochemical inflammatory symptoms and despite the absence of right atrial oedema on MRI. Currently, there are no predictors for reversibility of this rare myocarditis complication.

Myocarditis is being recognised as a potentially fatal complication of the H1N1 pandemic. This strain has a much higher myocardial affinity than the usual seasonal influenza virus. ${ }^{3}$ Patients with suspicion of $\mathrm{H} 1 \mathrm{~N} 1$ infection should be actively screened for cardiac conduction with ECG and Holter.

\section{Christian Steinberg, Santabhanu Chakrabarti}

Division of Cardiology, University of British Columbia, Vancouver, British Columbia, Canada

Correspondence to Dr Santabhanu Chakrabarti, Division of Electrophysiology, University of British Columbia at Vancouver-St. Paul's Hospital, 1081 Burrard St, Vancouver, British Columbia, Canada V6Z1Y6; chakrabartiss@gmail.com

Contributors CS and SC developed together the idea for the manuscript and wrote the manuscript.
Competing interests None.

Patient consent Obtained.

Provenance and peer review Not commissioned; externally peer reviewed.

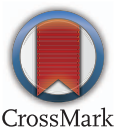

To cite Steinberg C, Chakrabarti S. Heart Asia 2015;7:16-17. doi:10.1136/heartasia-2014-010522

Heart Asia 2015;7:16-17. doi:10.1136/heartasia-2014-010522

\section{REFERENCES}

1 Beinart R, Morganti K, Ruskin J, et al. H1N1 influenza A virus induced atrioventricular block. J Cardiovasc Electrophysiol 2011;22:711-13.

2 Morimoto $S$, Kato S, Hiramitsu $S$, et al. Role of myocardial interstitial edema in conduction disturbances in acute myocarditis. Heart Vessels 2006;21:356-60.

3 Ukimura A, Satomi H, Ooi Y, et al. Myocarditis associated with influenza A H1N1pdm2009. Influenza Res Treat 2012;2012:351979. 